\title{
Initial Perceptions of a Touch-Based Tablet Handwriting Serious Game
}

\author{
Chek Tien Tan, Junbin Huang, and Yusuf Pisan \\ Games Studio, University of Technology, Sydney \\ \{chek, yusuf.pisan\}@gamesstudio.org
}

\begin{abstract}
This paper aims to evaluate a handwriting serious game that makes use of popular modern touch-based tablets to preserve traditional handwriting practice. A first playable prototype was built and a pilot study performed on an initial group of twenty participants. Significantly positive results were observed in the perceptions of usefulness and also across all gameplay dimensions except for flow.
\end{abstract}

Keywords: Serious Games, Player Experience, Handwriting.

\section{Introduction and Related Work}

In today's developed countries, people mostly communicate via electronic means and the practice of physically writing on pen and paper is getting rare. In young children, the problem of not getting enough handwriting practice is even more severe [13. However, even in this era of electronic communication dominance, handwriting still serves as an important cornerstone of basic language literacy. Individuals with clear and legible handwriting skills have been shown to have significantly better literacy skills [15].

Game-based learning is a mature domain that has demonstrated to be successful [14] but research in the area of handwriting games is however scarce. Maxim and Martineau [8] created a handwriting game for children on a tablet-PC and reported highly positive scores. However, the basis of their metrics was unclear and the description of their game mechanics did not portray a clear a level progression. They also mentioned that players are required to play mini-games in-between each handwriting task, which were possibly confounding variables that affected their results. The use of a hybrid tablet-PC meant for traditional keyboard and mouse inputs might not appeal to children as well.

In the related domain of handwriting rehabilitation, there is also some activity, though also minimal. Curtis et. al. 2] have created several mini-games for this purpose. However, it is targeted at patients with motor disorders with the goals of improving physical and mental health which are not the primary goals in this paper. Nevertheless, their qualitative evaluation have shown that all patients seemed to be motivated by the game premise and that direct feedback of their performance was an important feature of the system. 


\section{Penmanship Design and Evaluation}

Motivated by the above reasons, we create and evaluate a touch-based tablet handwriting serious game called Penmanship. As a first playable prototype, the goal was to keep the game as minimal as possible to demonstrate handwriting as the core gameplay mechanic. Having only one core mechanic to allows for a clear and concise test of the initial research questions we have set out to answer so that we can clearly correlate the responses to just the handwriting mechanic. A screenshot of the game can be seen in Figure 1.

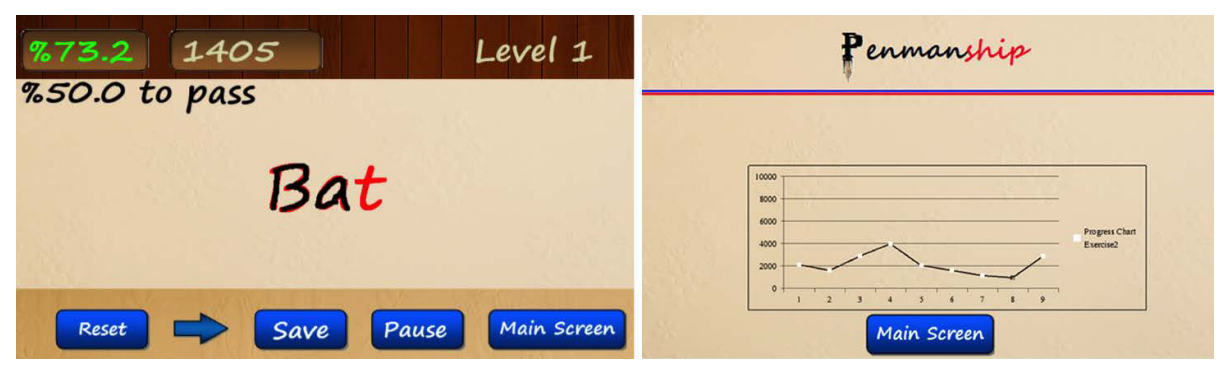

Fig. 1. Screenshots of the Penmanship game. The left picture shows the game state after a player tried to trace the word bat in a game level. Black lines indicate the player's trace and the red line represents the correct word template. Near the top left is a similarity percentage (updated in real-time so that player gets instant feedback) indicating the performance of the player in the current level, in terms of how well the trace matches the template. To the right of the percentage is a cumulative score for the player. The right picture shows the progress chart screen of the player's performance over time.

The game objective is simply to mimic word templates to reach a pre-set passing percentage in each level, in order to obtain as high a cumulative score as possible over time. Each successive level gets harder in terms of the word complexity, the similarity percentage required to conquer it, as well as the number of words required to clear a level. The gameplay premise was inspired by traditional handwriting tracing worksheets that we often see in the early years of education. The word templates were implemented based on the Segoe Print font, which closely resembles that of the New South Wales Foundation style handwriting 1 taught in all schools in New South Wales, Australia, where this research has taken place. Hence we hope to keep that traditional premise but with game elements added in the hopes of making it more engaging and fun.

As a pilot study, our goals were basically to find out initial perceptions on whether the game is useful for handwriting improvement and whether it provides a good gameplay experience even if it is a serious game. 20 participants (6 females) took part in the study with a mean age of 24 years $(S D=2.14)$. Penmanship was implemented in GameMaker Studio with the game compiled

${ }^{1}$ http://www.schoolfonts.com.au/Fonts_NSW.html 
on a Motorola Xoom MZ604 Android tablet and participants played using a capacitive stylus.

Participants completed a user survey performed after they have played for 20 to 25 minutes. The survey is based on the iGEQ [6] with an additional "usefulness" dimension for us to evaluate the perceptions on whether the game is useful to improve handwriting. A two-tailed one-sample t-test was performed against the midpoint average of 2.5 for the likert scores. This was because we wanted to find out whether each mean score was significantly different from merely an "on the fence" opinion. All the mean scores were significantly different $(p<0.05)$ except for the items "I felt content", "I felt good", "I found it impressive" and "Did you find Penmanship useful for improving your handwriting?". These insignificant responses were removed and the summarised mean scores for each dimension is shown in Figure 2. An unstructured interview was also conducted to obtain suggestions for improvement after they have played the game.

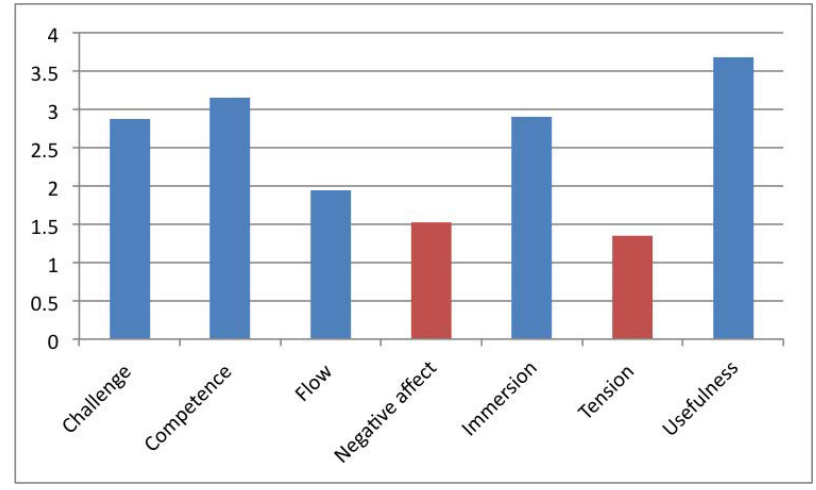

Fig. 2. Mean scores for each dimension in the survey. The y-axis represents the mean scores and the $\mathrm{x}$-axis represents each dimension. Positive affect is absent as the mean scores for both its constituent questions were statistically insignificant. Blue bars indicate positively phrased questions (higher is better) whilst red bars indicate negatively phrased questions (lower is better).

\section{Results and Conclusion}

The results show much promise for just a first playable prototype of Penmanship with a single handwriting mechanic as the core gameplay. From the summarised mean scores (Figure 2), it can be seen that the dimensions challenge, competence, immersion and usefulness means were significantly higher than average. This shows that players did experience these important gameplay experiences even with the minimalistic prototype we have made with just a single handwriting mechanic. The negatively phrased dimensions negative affect and tension also had means significantly lower than average which shows that players were not dismissive of the game. It should also be highlighted that the usefulness dimension had the highest mean score which gives us confidence on the "serious" aspect of the game. 
It can also be seen from Figure 2 that the flow dimension had a mean score that was significantly lower than average. Penmanship is built to be a casual game where players can stop and continue at anytime and hence is fundamentally different from highly immersive 3D games which focus more on providing a flow experience [1. Moreover, this was a first prototype which did not have the full level progression dynamics that contains more varied game mechanics.

Abundant feedback was also provided during the interviews. Many participants suggested that more varied challenges should be incorporated at higher levels. Other comments were mainly to improve the visual and aural aspects of the game in order to improve player feedback.

The feedback provided highlights our main limitation, which is the maturity of the gameplay elements. Although most dimensions were positive, scores were mostly modestly above average. Hence more work needs to be done on improving the implementation the game resources, the challenge progression, as well as adding premise, story and characters. These are hence left as future work.

\section{References}

1. Csikszentmihalyi, M.: Flow: The Psychology of Optimal Experience. P. S. Series, vol. 54. Harper \& Row (1990)

2. Curtis, J., Ruijs, L., de Vries, M.: Rehabilitation of handwriting skills in stroke patients using interactive games: A pilot study. In: CHI 2009 Extended Abstracts on Human Factors in Computing Systems, pp. 3931-3936 (2009)

3. Fullerton, T.: Game Design Workshop: A Playcentric Approach to Creating Innovative Games. Elsevier Morgan Kaufmann (2008)

4. Gee, J.P.: What video games have to teach us about learning and literacy. Computers in Entertainment 1(1), 20 (2003)

5. Graham, S.: Want to Improve Children's Writing? American Educator, 20-28 (2010)

6. IJsselsteijn, W.A., De Kort, Y.A.W., Poels, K.: The Game Experience Questionnaire: Development of a self-report measure to assess the psychological impact of digital games. Technical report, TU Eindhoven (2007)

7. Jeffries, S.: The death of handwriting. The Guardian (2006)

8. Maxim, B.R., Martineau, N.D.: Running head- Learning via Gaming- An Immersive Environment for Teaching Handwriting. Meaningful Play, pp. 1-12 (2008)

9. McGregor, R., Meiers, M.: Writing K-12: A guide to assessment \& reporting. English Club (1987)

10. Michael, D., Chen, S.: Serious games: Games that educate, train, and inform. vol. 1. Thomson Course Technology (2006)

11. Nacke, L.E.: Affective Ludology, vol. 2. Blekinge Institute of Technology (2009)

12. Prensky, M.: Digital Game-Based Learning. Paragon House (2007)

13. Sheffield, B.: Handwriting: A neglected cornerstone of literacy. Annals of Dyslexia 46(1), 21-35 (1996)

14. Susi, T., Johannesson, M., Backlund, P.: Serious games: An overview. Institutionen för kommunikation och information (2007)

15. Wray, D., Medwell, J.: The links between handwriting and composing in primary aged children. Cambridge Journal of Education 39(3), 329-344 (2009) 